\title{
ON THE EXISTENCE OF THE BIHARMONIC GREEN KERNELS AND THE ADJOINT BIHARMONIC FUNCTIONS
}

\author{
ABDERRAHIM ASLIMANI, IMAD EL GHAZI, MOHAMED EL KADIRI, \\ AND SABAH HADDAD
}

\begin{abstract}
We study the existence and the regularity of the biharmonic Green kernel in a Brelot biharmonic space whose associated harmonic spaces have Green kernels. We show by some examples that this kernel does not always exist. We then introduce and study the adjoint of the given biharmonic space. This study was initiated by Smyrnelis, however, it seems that several results were incomplete and we clarify them here.
\end{abstract}

\section{INTRODUCTION}

The study of the Laplace equation $\Delta u=0$ on an open subset $U$ of $\mathbb{R}^{n}$ led to various axiomatic theories of harmonic functions (Brelot, Bauer, ConstantinescuCornea and Doob) that apply more generally to partial differential equations of the type $L u=0$, where $L$ is an elliptic or parabolic second-order linear differential operator. Such theories do not apply to the equations of higher order as the classical biharmonic equation $\Delta^{2} u=\Delta(\Delta u)=0$ in $U$ or, equivalently, to the system $\Delta u=-v, \Delta v=0$. This led Smyrnelis to develop and study in [16, 17] an axiomatic theory within a larger framework which apply to the partial differential equations $L_{1} L_{2} u=0$, where $L_{1}$ and $L_{2}$ are two elliptic or parabolic second-order linear differential operators on an open subset of $\mathbb{R}^{n}$ or of a Riemannian manifold. In this theory, a biharmonic space is the data of a locally compact space $\Omega$ with a sheaf $\mathcal{H}$ of vector spaces of pairs of continuous real functions (called the biharmonic pairs) satisfying certain axioms (cf. Section 2). To such space are associated two harmonic spaces $\left(\Omega, \mathcal{H}_{1}\right)$ and $\left(\Omega, \mathcal{H}_{2}\right)$ (cf. [16, Theorem 1.29, p. 57]). Then one introduces the superharmonic and potential pairs and extends to this framework the notion of balayage, Dirichlet problem and other concepts of classical or axiomatic theories of harmonic functions. If $L_{1}$ and $L_{2}$ are two elliptic or parabolic second-order linear differential operators on an open subset $\Omega$ of $\mathbb{R}^{n}$, then $\Omega$ endowed with the sheaf $\mathcal{H}$ on

2000 Mathematics Subject Classification. 31B30, 31C40, 31D05.

Key words and phrases. Harmonic function, Biharmonic function, Harmonic space, Biharmonic space, Green kernel, Potential, Biharmonic Green kernel, Adjoint harmonic function, Adjoint biharmonic function. 
$\Omega$ defined by $\mathcal{H}(U)=\left\{(u, v) \in \mathcal{C}^{2}(U): L_{1} u=-v, L_{2} v=0\right\}$ for every open subset $U$ of $\Omega$, is a biharmonic space in the sense of Smyrnelis.

Let $(\Omega, \mathcal{H})$ be a strong biharmonic space (that is, there is a $\mathcal{H}$-potential $(p, q)>0$ on $\Omega)$ whose associated harmonic spaces $\left(\Omega, \mathcal{H}_{1}\right)$ and $\left(\Omega, \mathcal{H}_{2}\right)$ are Brelot spaces. We assume that the $\mathcal{H}_{2}$-potentials of the same harmonic support reduced to one point (that is, $\mathcal{H}_{2}$-harmonic outside a set reduced to a single point) are proportional. According to [14, Proposition 22.1, p. 507] there exists a continuous function $y \mapsto q_{y}$ from $\Omega$ into a compact base $B$ of the cone $\mathcal{S}_{2}^{+}(\Omega)$ of $\mathcal{H}_{2}$-superharmonic functions $\geq 0$ on $\Omega$ endowed with the topology of R-M. Hervé cf. [14, Chap. IV]. The function $G$ defined on $\Omega^{2}=\Omega \times \Omega$ by $G(x, y)=q_{y}(x)$ is l.s.c. on $\Omega^{2}$ and continuous outside the diagonal of $\Omega^{2}(G$ will be called a (or the) Green kernel of the harmonic space $\left(\Omega, \mathcal{H}_{2}\right)$ ). Under the assumption $(1,1) \in \mathcal{H}^{*}(\Omega)$ Smyrnelis affirms in [20] that, for every $y \in \Omega$, the pure hyperharmonic function $p_{y}^{\prime}$ of order 2 associated with $q_{y}$ (see Section 5]) is $\mathcal{H}_{1}$-superharmonic function and that the function $y \mapsto p_{y}^{\prime}$ is continuous from $\Omega$ into $\mathcal{S}_{1}^{+}(\Omega)$. This result was used by Smyrnelis to define in [21] adjoint biharmonic functions. It is also stated in a remark at the end of [20], that there is a biharmonic pair $(h, k)$ such that $h>0$ and $k>0$ on $\Omega$. By several examples stated in Section 5 below, we see that these statements are not always true, and therefore the results of [21] are incomplete without additional assumptions ensuring the existence of a biharmonic Green kernel (or of a second Green kernel). In Section 6 of the present work we also consider adjoint biharmonic functions. We then define the adjoint biharmonic space $\left(\Omega,{ }^{*} \mathcal{H}\right)$ of $(\Omega, \mathcal{H})$ under the following supplementary conditions:

1. For any $y \in \Omega$, the pure hyperharmonic function $p_{y}^{\prime}$ of order two associated with $q_{y}$ is $\mathcal{H}_{1}$-superharmonic,

2. For any $x \in \Omega$, the function $y \mapsto p_{y}^{\prime}(x)$ is continuous on $\Omega \backslash\{x\}$.

It is a biharmonic space whose associated harmonic spaces are respectively the adjoint harmonic spaces $\left(\Omega,{ }^{*} \mathcal{H}_{2}\right)$ and $\left(\Omega,{ }^{*} \mathcal{H}_{1}\right)$ of the harmonic spaces $\left(\Omega, \mathcal{H}_{2}\right)$ and $\left(\Omega, \mathcal{H}_{1}\right)$ respectively, and in which the pure hyperharmonic function of order 2 associated with $p_{y}^{*}$ is the function $p_{y}^{\prime *}=p^{\prime}(y)$ for every $y \in \Omega$, where $y \mapsto p_{y}$ is a continuous map from $\Omega$ into $\mathcal{S}_{1}^{+}(\Omega)$ which associates to any $y \in \Omega$ an $\mathcal{H}_{1}$-potential with harmonic support $\{y\}$. We also show that, conversely, if there is a biharmonic space $(\Omega, \mathcal{G})$ whose associated biharmonic spaces are $\left(\Omega,{ }^{*} \mathcal{H}_{2}\right)$ and $\left(\Omega,{ }^{*} \mathcal{H}_{1}\right)$ respectively, and where the pure hyperharmonic function of order 2 associated with $p_{y}^{*}$ is the function $p_{y}^{*}=p^{\prime}(y)$ for every $y \in \Omega$ (where $p_{y}^{*}$ is the $\mathcal{H}_{1}$-adjoint superhharmonic defined by $p_{y}(x)=p_{x}(y)$ ), then the above condition $\mathbf{2}$ is satisfied and the coupling kernel of $(\Omega, \mathcal{G})$ is equal to the adjoint kernel $V^{*}$ of the coupling kernel $V$ of $(\Omega, \mathcal{G})$ (see definition of 
$V^{*}$ in Section 6). We also show that the (function) kernel $H$ defined on $\Omega^{2}$ by $H(x, y)=p_{y}^{\prime}(x)$, called the biharmonic Green kernel (or the second Green kernel) of the biharmonic space $(\Omega, \mathcal{H})$, is regular in the sense that it is l.s.c. on $\Omega^{2}$ and continuous outside the diagonal of $\Omega^{2}$.

In this paper we also give results in the axiomatic theory of biharmonic spaces and clarify some incomplete results of Smyrnelis. More precisely, we shall prove the following:

1. In a biharmonc space associated with Green harmonic space the biharmonic Green kernel may not exist.

2. The existence of biharmonic space associated with two harmonic spaces and a given coupling kernel.

3. Contrarily to the case of harmonic space, a biharmonic pair $>0$ (or a strict biharmonic function $>0$ ) in a strong biharmonic space may not exist.

4. A definition of the notion of biharmonic adjoint of given Green biharmonic space is possible.

5. The biharmonic Green kernel in a biharmonic space, when it exists, is regular under some simple conditions.

Notations. Let $\Omega$ be a locally compact space with countable base. If $A$ is a subset of $\Omega$, we denote by $\bar{A}$ the closure of $A$ in the Alexandroff compactification $\bar{\Omega}$ of $\Omega$, and by $\partial A$ the boundary of $A$ in $\bar{\Omega}$. By function on $A$ we mean a function on $A$ with values in $[-\infty,+\infty]$. Let $U$ be an open subset of $\Omega$, the set of Borel functions on $U$ is denoted by $\mathcal{B}(U)$ and, if $f$ is a function defined on $U$, we denote by $\widehat{f}$ the l.s.c. regularization of $f$. Recall that $\widehat{f}$ is defined by $\widehat{f}(x)=\liminf _{y \rightarrow x} f(y)$ for every $x \in U$ and that $\widehat{f}$ is the greatest 1.s.c. of $f$ on $U$. We denote by $\mathcal{C}_{c}(\Omega)$ the (real) vector space of continuous and finite functions on $\Omega$ with compact support. For every continuous function $\varphi$ on $\Omega$, we denote by $S(\varphi)$ the support of $\varphi$. The order on the set of pairs of functions on a subset $A$ of $\Omega$ is defined by

$$
\left(f_{1}, g_{1}\right) \geq\left(f_{2}, g_{2}\right) \Longleftrightarrow f_{1} \geq f_{2} \text { et } f_{2} \geq g_{2} \text { on } A \text {. }
$$

For the sake of simplicity, we shall write $(f, g) \geq 0$ for $(f, g) \geq(0,0)$ and $(f, g)>0$ on $A$ for $f$ and $g>0$ on $A$.

If $\mathcal{A}(E)$ is a set of functions defined on a set $E$, we denote by $\mathcal{A}^{+}(E)$ the set of nonnegative functions of $\mathcal{A}(E)$.

\section{Preliminaries And notations}

Throughout this article, $\Omega$ is a locally compact space with countable base and $\mathcal{U}$ (resp. $\mathcal{U}_{c}$ ) is the set of open subsets (resp. the set of relatively compact open subsets) of $\Omega$. Let $\mathcal{H}$ be a map which associates to each $U \in \mathcal{U}$ a vector space of pairs of continuous real functions on $U$, compatible in the sense that 
if $(u, v) \in \mathcal{H}(U)$ and $u=0$ on an open subset $\omega \subset U$ then $v=0$ on $\omega$. The pairs of $\mathcal{H}(U)$ are termed biharmonic on $U$.

Axiom 1. $\mathcal{H}$ is a sheaf.

This means that

1. If $U$ and $V$ are both open subsets of $\Omega$ such that $U \subset V$ and if $(u, v) \in$ $\mathcal{H}(V)$ then $\left(u_{\mid U}, v_{\mid U}\right) \in \mathcal{H}(U)$.

2. If $\left(U_{i}\right)$ is a family of open subsets of $\Omega$ and if $(u, v)$ is a pair of real functions on $U=\bigcup_{i} U_{i}$ such that, for every $i,\left(u_{\mid U_{i}}, v_{\mid U_{i}}\right) \in \mathcal{H}\left(U_{i}\right)$, then $(u, v) \in \mathcal{H}(U)$.

An open subset $\omega \in \mathcal{U}_{c}$ is said to be $\mathcal{H}$-regular, or just regular, if for every pair $(f, g)$ of real continuous functions on $\partial \omega$, there exists a pair $(u, v) \in \mathcal{H}(\omega)$ such that

i) $\lim _{x \rightarrow y} u(x)=f(y)$ and $\lim _{x \rightarrow y} v(x)=g(y)$ for every $y \in \partial \omega$.

ii) If $(f, g) \geq(0,0)$, then $(u, v) \geq(0,0)$.

Such pair is then unique. It is denoted by $H_{\omega}(f, g)=\left(H_{\omega}^{1}(f, g), H_{\omega}^{2}(f, g)\right)$ and called the solution of the Riquier problem in $\omega$ for the boundary data $(f, g)$ on $\partial \omega$. If $\omega \in \mathcal{U}_{c}$ is $\mathcal{H}$-regular, then for any $x \in \omega$, there exists a triple $\left(\mu_{x}^{\omega}, \nu_{x}^{\omega}, \lambda_{x}^{\omega}\right)$ of nonnegative Radon measures on $\partial \omega$, called the triple of biharmonic measures of $\omega$ at the point $x$, such that

$$
H_{\omega}^{1}(f, g)(x)=\int f(y) d \mu_{x}^{\omega}(y)+\int g(y) d \nu_{x}^{\omega}(y)
$$

and

$$
H_{\omega}^{2}(f, g)(x)=\int g(y) d \lambda_{x}^{\omega}(y)
$$

for any pair $(f, g) \in \mathcal{C}(\partial \omega) \times \mathcal{C}(\partial \omega)$.

Axiom 2. The regular open sets form a base of the topology of $\Omega$.

We denote by $\mathcal{U}_{r}$ the set of all $\mathcal{H}$-regular relatively compact open subsets of $\Omega$. A pair $(u, v)$ of l.s.c. functions on $U \in \mathcal{U}$ with values in $]-\infty,+\infty]$ is said to be $\mathcal{H}$-hyperharmonic (on $U$ ), or just hyperharmonic when there is no risk of confusion, if for any $\omega \in \mathcal{U}_{r}, \omega \subset \bar{\omega} \subset U$, and any $x \in \omega$, we have

$$
\int^{*} u d \mu_{x}^{\omega}+\int^{*} v d \nu_{x}^{\omega} \leq u(x)
$$

and

$$
\int^{*} v d \lambda_{x}^{\omega} \leq v(x)
$$


The set of $\mathcal{H}$-hyperharmonic pairs on an open subset $U$ of $\Omega$ is denoted by $\mathcal{H}^{*}(U)$. It is easy to check that $\mathcal{H}^{*}(U)$ and $\mathcal{H}^{*+}(U)$ are convex cones.

For every $U \in \mathcal{U}$ we set

$$
\mathcal{H}_{1}^{*}(U)=\left\{u:(u, 0) \in \mathcal{H}^{*}(U)\right\}
$$

and

$$
\mathcal{H}_{2}^{*}(U)=\left\{v:(+\infty, v) \in \mathcal{H}^{*}(U)\right\} .
$$

Then $\mathcal{H}_{1}^{*}$ and $\mathcal{H}_{2}^{*}$ are two sheaves of cones of l.s.c. functions.

Then we define on $\Omega$ two sheaves of vector spaces of continuous real functions by putting $\mathcal{H}_{1}(U)=\mathcal{H}_{1}^{*}(U) \cap\left(-\mathcal{H}_{1}^{*}(U)\right)$ and $\mathcal{H}_{2}(U)=\mathcal{H}_{2}^{*}(U) \cap\left(-\mathcal{H}_{2}^{*}(U)\right)$ for any open subset $U$ of $\Omega$.

Axiom 3. $\left(\Omega, \mathcal{H}_{1}\right)$ and $\left(\Omega, \mathcal{H}_{2}\right)$ are Bauer harmonic spaces.

We say that the pair $(\Omega, \mathcal{H})$ or that $\Omega$, provided with the sheaf $\mathcal{H}$, is a biharmonic space if the axioms 1,2 and 3 are satisfied.

Example 2.1. Let $N$ be an integer $\geq 1$, and let $L_{1}, L_{2}$ two elliptic or parabolic second-order linear differential operators, on an (nonempty) open subset $\Omega$ of $\mathbb{R}^{N}$. Then $\Omega$ endowed with the sheaf $\mathcal{H}$ defined by

$$
\mathcal{H}(U)=\left\{(h, k) \in \mathcal{C}^{2}(U) \times \mathcal{C}^{2}(U): L_{1} h=-k, L_{2} k=0\right\}
$$

for every open subset $U$ of $\Omega$, is a biharmonic space.

A biharmonic space $(\Omega, \mathcal{H})$ is said to be a Brelot biharmonic space if $\Omega$ is non-compact, connected and locally connected and if the associated harmonic spaces $\left(\Omega, \mathcal{H}_{1}\right)$ and $\left(\Omega, \mathcal{H}_{2}\right)$ are Brelot harmonic spaces.

The probabilistic aspect of biharmonic spaces was studied by Bouleau in [3]. Boboc and Bucur [2] have shown that the nonnegative hyperharmonic pairs can be identified with the excessive functions of a triangular resolvent on the space $\Omega \oplus \Omega$, and then the theory of biharmonic spaces reduces to that of $H$-cones. For more details on the theory of biharmonic spaces, we refer to [16] and [17].

Let $U \in \mathcal{U}$ and $(u, v) \in \mathcal{H}^{*}(U)$. If $u$ is finite on a dense subset in $U$ (and hence $v$ also is finite in a dense subset in $U$ ), we say that the pair $(u, v)$ is $\mathcal{H}$-superharmonic or just superharmonic on $U$. We denote by $\mathcal{S}(U)$ the set of all superharmonic pairs on $U$. Then $\mathcal{S}(U), \mathcal{S}^{+}(U)$ and $\mathcal{H}^{+}(U)$ are convex cones. 
Let $(u, v)$ be a $\mathcal{H}$-superharmonic pair on $\Omega$. It follows from Axiom 1 that there is a greatest open subset $U$ of $\Omega$ on which $(u, v)$ is $\mathcal{H}$-harmonic. The complement set of $U$ is called the biharmonic support of $(u, v)$.

A pair $(p, q) \in \mathcal{S}^{+}(\Omega)$ is said to be a $\mathcal{H}$-potential (or just a potential if there is no risk of confusion) if

$$
\forall(h, k) \in \mathcal{H}^{+}(\Omega) ;(h, k) \leq(p, q) \Rightarrow h=k=0 .
$$

It is easy to see that if $(u, v) \in \mathcal{S}^{+}(\Omega)$ and if $u$ is a $\mathcal{H}_{1}$-potential and $v$ is a $\mathcal{H}_{2}$-potential, then $(u, v)$ is a $\mathcal{H}$-potential. Conversely, if $(u, v)$ is a $\mathcal{H}$-potential, then $u$ is a $\mathcal{H}_{1}$-potential and, as it will be seen in the next section (see Remark 3.9), $v$ is a $\mathcal{H}_{2}$-potential.

A biharmonic space $(\Omega, \mathcal{H})$ is said to be strong, if there exists a $\mathcal{H}$-potential $(p, q)>0$ on $\Omega$. For $L_{1}=L_{2}=\Delta$, the Laplace operator, in the Example 2.1, the space $\left(\mathbb{R}^{N}, \mathcal{H}\right)$ is strong if and only if $N \geq 5$ (see [10]). Any relatively compact open subset $\omega$ of a biharmonic space, with the biharmonic sheaf restricted to $\omega$, is strong.

In order to simplify some statements in the next sections we introduce the following definition:

Definition 2.2. An harmonic space $(\Omega, \mathcal{K})$ is called a Green harmonic space if $(\Omega, \mathcal{K})$ is a Brelot harmonic space satisfying the hypothesis of uniqueness, that is, the potentials on $\Omega$ with the same harmonic support reduced to one point are proportional. A biharmonic space $(\Omega, \mathcal{H})$ is called Green biharmonic space if its associated harmonic spaces $\left(\Omega, \mathcal{H}_{1}\right)$ and $\left(\Omega, \mathcal{H}_{2}\right)$ are Green harmonic spaces.

\section{Pure hyperharmonic pairs and kernels associated With a BIHARMONIC SPACE}

Let $(\Omega, \mathcal{H})$ be a biharmonic space and $v$ a nonnegative $\mathcal{H}_{2}$-hyperharmonic function on $\Omega$. The set

$$
\mathcal{U}_{0}(v)=\left\{u \in \mathcal{H}_{1}^{*+}(\Omega):(u, v) \in \mathcal{H}_{+}^{*}(\Omega)\right\}
$$

is not empty because the constant function $u \equiv+\infty$ belongs to $\mathcal{U}_{0}(v)$. Then, according to [17, Lemma 11.6, p. 36] the function $u_{0}=\widehat{\inf } \mathcal{U}_{0}(v) \in \mathcal{H}_{1}^{*+}(\Omega)$. It is the smallest nonnegative $\mathcal{H}_{1}$-hyperharmonic function $u$ on $\Omega$ such that $(u, v) \in \mathcal{H}^{*+}(\Omega)$. This function is called the pure hyperharmonic function of order 2 , or simply the pure $\mathcal{H}_{1}$-hyperharmonic, associated with $v$. A pair $(u, v) \in \mathcal{H}^{*+}(\Omega)$ is said to be pure if $u$ is the pure hyperharmonic function of order 2 associated with $v$.

The essential properties of the pure hyperharmonic pairs can be found in [3] and 4. 
Remark 3.1. If the pair $(u, v) \in \mathcal{S}^{+}(\Omega)$ is pure, then $u$ is a $\mathcal{H}_{1}$-potential. Indeed, $u$ is nonnegative $\mathcal{H}_{1}$-superharmonic. Let $h$ be the $\mathcal{H}_{1}$-harmonic part in the Riesz decomposition of $u$, then the pair $(u-h, v)$ is nonnegative $\mathcal{H}$ superharmonic in $\Omega$, whence $u \leq u-h$ and therefore $h=0$, which clearly proves that $u$ is a $\mathcal{H}_{1}$-potential.

Remark 3.2. Let $(p, q)$ be a pure $\mathcal{H}$-superharmonic pair on $\Omega$ with $q>0$, then $p$ is a strict potential. In fact, for any $\omega \in \mathcal{U}_{r}$, we have $p \geq \int p d \mu^{\omega}$. $+\int q d \nu^{\omega}$. $>$ $\int p d \mu_{.}^{\omega}$. Thus $p$ is strict.

Recall that a kernel $V$ on a measurable space $(E, \mathcal{E})$ is a function $V \geq 0$ defined on $E \times \mathcal{E}$ such that

1. For every $A \in \mathcal{E}$, the function $x \mapsto V(x, A)$ is $\mathcal{E}$-measurable.

2. For every $x \in E$, the set function $A \mapsto V(x, A)$ is a (nonnegative) measure on $(E, \mathcal{E})$.

Let $V$ be a kernel on $(E, \mathcal{E})$, then for any nonnegative $\mathcal{E}$-measurable function $f$ on $E$, we denote by $V f$ or $V(f)$ the function on $E$ defined by

$$
V f(x)=\int f(y) V(x, d y), \forall x \in E,
$$

where the integral is understood to be taken with respect to the measure $V(x, \cdot)$.

Let $\mu$ be a measure on $(E, \mathcal{E})$ and $f$ a nonnegative $\mathcal{E} \otimes \mathcal{E}$-measurable function on $E \times E$, then the function $(x, A) \mapsto \int_{A} f(x, y) d \mu(y)$ on $E \times \mathcal{E}$ is a kernel on $(E, \mathcal{E})$.

If $E$ is a topological space and $\mathcal{E}$ is the Borel $\sigma$-algebra of $E$, then a kernel on $(E, \mathcal{E})$ is called a Borel kernel on $E$.

Recall the following result of Bouleau [3, Theorem 2.8, p. 208] that will be very useful in the rest this paper:

Theorem 3.3. Let $(\Omega, \mathcal{H})$ be a strong biharmonic space. There exists a unique Borel kernel $V$ on $\Omega$ such that

1. For any function $\varphi \in \mathcal{C}_{c}^{+}(\Omega)$, the function $V \varphi$ is a $\mathcal{H}_{1}$-potential on $\Omega$, and $\mathcal{H}_{1}$-harmonic on $\Omega \backslash S(\varphi)$.

2. For every function $v \in \mathcal{H}_{2}^{*+}(\Omega), V v$ is the pure hyperharmonic function of order 2 associated with $v$.

The kernel $V$ will be called the coupling kernel of the harmonic spaces $\left(\Omega, \mathcal{H}_{1}\right)$ and $\left(\Omega, \mathcal{H}_{2}\right)$ in the biharmonic space $(\Omega, \mathcal{H})$ or simply the coupling kernel of $(\Omega, \mathcal{H})$.

Let $(p, q)$ be a pure finite and continuous $\mathcal{H}$-potential $>0$ on $\Omega$. We have seen in the Remark 3.2 that the potential $p$ is strict. Then there exists by $[8$, 
Exercice 8.2.3, p. 198] (or [15, Theorem 2, p. 362] for Brelot harmonic spaces) a unique Borel kernel $W$ on $\Omega$ such that

1. For any function $\varphi \in \mathcal{C}_{c}^{+}(\Omega)$, the function $W \varphi$ is an $\mathcal{H}_{1}$-potential on $\Omega$, and $\mathcal{H}_{1}$-harmonic on $\Omega \backslash S(\varphi)$.

2. $W 1=p$.

Let $V$ be the coupling kernel of $(\Omega, \mathcal{H})$, and consider the Borel kernel $V^{\prime}$ on $\Omega$ defined by $V^{\prime} f=V(q f)$ for any Borel function $f \geq 0$ on $\Omega$. Then $V^{\prime}$ satisfies the above two properties 1 . and 2. of $W$. Hence, according to the uniqueness of the kernel $W$, we have $W=V^{\prime}$. From this result we deduce the following:

Proposition 3.4. For every $\mathcal{H}_{2}$-hyperharmonic function $v \geq 0$ on $\Omega$, the pure $\mathcal{H}_{2}$-hyperharmonic function associated with $v$ is $u=W\left(\frac{v}{q}\right)$.

Proposition 3.5. Let $(u, v) \in \mathcal{S}_{+}^{*}(\Omega)$ and let $u_{0}$ be the pure $\mathcal{H}_{1}$-hyperharmonic function of order 2 associated with $v$. Then there exists a nonnegative $\mathcal{H}_{1}$ hyperharmonic function $u_{1}$ in $\Omega$ such that $u=u_{0}+u_{1}$.

Proof. Since $(u, v)$ is $\mathcal{H}$-superharmonic then $u$ and $u_{0}$ are $\mathcal{H}_{1}$-superharmonic functions. Suppose first that $u$ and $v$ are finite and let $\omega \in \mathcal{U}_{r}$ be a $\mathcal{H}$-regular open subset and let $w$ be the function defined on $\omega$ by $w=u-\int u d \mu^{\omega}+$ $\int u_{0} d \mu_{.}^{\omega}$. Then the pair $(w, v)$ is $\mathcal{H}$-superharmonic on $\omega$. Furthermore we have $\liminf _{x \in \omega, x \rightarrow z} w(x) \geq u_{0}(z)$ for every $z \in \partial \omega$. It therefore follows from [16, Proposition 1.21, p. 53] that the pair $\left(u_{2}, v\right)$ is $\mathcal{H}$-superharmonic in $\Omega$, where $u_{2}$ is the function defined on $\Omega$ by

$$
u_{2}=\left\{\begin{array}{c}
w \wedge u_{0} \text { on } \omega \\
u_{0} \text { on } \Omega \backslash \omega
\end{array}\right.
$$

hence $u_{2} \geq u_{0}$ and therefore $w \geq u_{0}$ in $\omega$. Then it follows that the nonnegative function $u-u_{0}$ is $\mathcal{H}_{1}$-hyperharmonic function $u_{1}$ on $\Omega$ and we have $u=u_{0}+u_{1}$. For general $\mathcal{H}$-hyperharmonic pair $(u, v)$, there exists an increasing sequence $\left(\left(u_{n}, v_{n}\right)\right)$ of finite $\mathcal{H}$-superharmonic pairs such that $(u, v)=\sup _{n}\left(u_{n}, v_{n}\right)$. By the above case, for each $n$ there exists a $\mathcal{H}_{1}$-superharmonic $t_{n}$ and a pure pair $\left(u_{n}^{\prime}, v_{n}\right)$ such that $u_{n}=u_{n}^{\prime}+t_{n}$. By Theorem 3.3, the sequence $\left(\left(u_{n}^{\prime}, v_{n}\right)\right)$ is increasing and the pair $\left(\sup _{n} u_{n}^{\prime}, v\right)$ is pure. It follows that $u=\sup _{n} u_{n}^{\prime}+$ $\sup _{m} \widehat{\inf }_{n \geq m} t_{m}$, which proves the proposition.

Corollary 3.6. Suppose that the biharmonic space $(\Omega, \mathcal{H})$ is strong. Then there exists a pure finite and continuous $\mathcal{H}$-potential $(p, q)>0$ on $\Omega$.

Proof. By [16, Proposition 7.6, p. 3], there exists a finite continuous $\mathcal{H}$ potential pair $(p, q)>0$ on $\Omega$. Let $p_{1}$ be the pure $\mathcal{H}_{1}$-hyperharmonic function associated with $q$, there exists by the preceding proposition a $\mathcal{H}_{1}$-superharmonic function $u \geq 0$ such that $p=u+p_{1}$. Since $u$ and $p_{1}$ are l.s.c. and $p$ is finite and continuous on $\Omega$, we deduce that $p_{1}$ is finite and continuous, and therefore $\left(p_{1}, q\right)$ is a pure finite and continuous $\mathcal{H}$-potential on $\Omega$. 
Corollary 3.7. Assume that $(\Omega, \mathcal{H})$ is a strong biharmonic space and let $(u, v)$ be pure pair on $\Omega$. Then there exists an increasing sequence $\left(\left(u_{n}, v_{n}\right)\right)$ of pure finite and continuous $\mathcal{H}$-potentials such that $(u, v)=\sup _{n}\left(u_{n}, v_{n}\right)$.

Proof. By [17, Théorème 7.8, p. 4], there exists an increasing sequence $\left(p_{n}, q_{n}\right)$ of continuous $\mathcal{H}$-potentials on $\Omega$ such that $(u, v)=\sup _{n}\left(p_{n}, q_{n}\right)$. For each $n$ let $p_{n}^{\prime}$ be the pure $\mathcal{H}_{1}$-hyperharmonic function associated with $q_{n}$. Then by Proposition 3.5 there exists $t_{n} \in \mathcal{H}_{1}^{*+}(\Omega)$ such $p_{n}=t_{n}+p_{n}^{\prime}$. Since $p_{n}$ is continuous and that $t_{n}$ and $p_{n}^{\prime}$ are l.s.c., then $p_{n}^{\prime}$ is continuous. Moreover, it follows from Theorem 3.3 that the sequence $\left(p_{n}^{\prime}\right)$ is increasing and $(u, v)=$ $\sup _{n}\left(p_{n}^{\prime}, q_{n}\right)$.

Proposition 3.8. Let $(u, v) \in \mathcal{S}^{+}(\Omega)$ be a pure pair and $U$ an open subset of $\Omega$. If $v$ is $\mathcal{H}_{2}$-harmonic on $U$, then the pair $(u, v)$ is $\mathcal{H}$-harmonic on $U$.

Proof. Let $\omega \in \mathcal{U}_{r}$ such that $\bar{\omega} \subset U$. Then we have $\int v d \lambda_{x}^{\omega}=v(x)$ for every $x \in \omega$, hence the pair

$$
(s, v)=\left\{\begin{array}{c}
\left(\int u d \mu_{.}^{\omega}+\int v d \nu_{.}^{\omega}, v\right) \text { on } \omega \\
(u, v) \text { on } \Omega \backslash \omega
\end{array}\right.
$$

is $\mathcal{H}$-hyperharmonic on $\Omega$ by [16, Proposition 4.2, p. 73]. Hence $s \geq u$ and therefore $u=s=\int u d \mu^{\omega}+\int v d \nu^{\omega}$. on $\omega$. Since $\omega$ is arbitrary, this proves that the pair $(u, v)$ is biharmonic on $U$ by [16, Proposition 5.4, p. 76].

Corollary 3.9. If a pair $(u, v) \in \mathcal{S}^{+}(\Omega)$ is a $\mathcal{H}$-potential, then $v$ is a $\mathcal{H}_{2}-$ potential.

Proof. The function $v$ being $\mathcal{H}_{2}$-superharmonic, let $k$ be the $\mathcal{H}_{2}$-harmonic part of $v$ in its Riesz decomposition and $h$ the pure $\mathcal{H}_{2}$-hyperharmonic associated with $k$. Then $h \leq u$, hence $(h, k)$ is $\mathcal{H}$-superharmonic and therefore biharmonic by the above proposition. It follows that $(h, k)=0$, and hence $k=0$, which proves that $v$ is a $\mathcal{H}_{2}$-potential.

Corollary 3.10. Let $(h, k)$ be a nonnegative $\mathcal{H}$-harmonic pair and let $p$ be the $\mathcal{H}_{1}$-potential part of the Riesz decomposition of the $\mathcal{H}_{1}$-superharmonic function $h$. Then $(p, k)$ is a pure $\mathcal{H}$-biharmonic pair.

Let $(\Omega, \mathcal{H})$ be a biharmonic space and suppose that the harmonic space $\left(\Omega, \mathcal{H}_{1}\right)$ is a Green space. Consider a continuous function $y \mapsto p_{y}$ from $\Omega$ into the cone $\mathcal{S}_{1}^{+}(\Omega)$ endowed with the topology of R-M. Hervé, which associates to any $y \in \Omega$ a $\mathcal{H}_{1}$-potential of harmonic support $\{y\}$ (cf. [14, Chap. IV] and [14, Proposition 22.1, p. 507]). Hence, according to the theorem of integral representation of potentials (cf. [14, Theorem 18.2, p. 482]), there exists a unique nonnegative Radon measure $\mu$ on $\Omega$ such that $p=\int p_{y} d \mu(y)$. In this 
case the kernel $W$ is then given by

$$
W f(x)=\int_{\Omega} p_{y}(x) f(y) d \mu(y)
$$

for any $x \in \Omega$ and any function $f \in \mathcal{B}^{+}(\Omega)$. As it can be seen from the above considerations, the kernel $V$ of Theorem 3.3 is given by

$$
V f(x)=\int_{\Omega} \frac{p_{y}(x)}{q(y)} f(y) d \mu(y)
$$

for any $x \in \Omega$ and any function $f \in \mathcal{B}^{+}(\Omega)$. Thus we have established the following proposition:

Proposition 3.11. Let $(\Omega, \mathcal{H})$ be a strong biharmonic space and suppose that the associated harmonic space $\left(\Omega, \mathcal{H}_{1}\right)$ is a Green space and with coupling kernel $V$. Consider a continuous function $y \mapsto p_{y}$ from $\Omega$ into the cone $\mathcal{S}_{1}^{+}(\Omega)$ of $\mathcal{H}_{1}$-superharmonic functions $\geq 0$ endowed with the topology of $R-M$. Hervé, associating to any $y \in \Omega$ a $\mathcal{H}_{1}$-potential of harmonic support $\{y\}$. Then there exists a unique Radon measure $\mu \geq 0$ on $\Omega$ such that $V f(x)=\int p_{y}(x) f(y) d \mu(y)$ for any $x \in \Omega$ and any $f \in \mathcal{B}^{+}(\Omega)$.

\section{BIHARMONIC SPACE ASSOCIATED WITH TWO STRONG HARMONIC SPACES AND A GIVEN COUPLING KERNEL}

Given two harmonic spaces $\left(\Omega, \mathcal{H}_{1}\right)$ and $\left(\Omega, \mathcal{H}_{2}\right)$, the first space assumed to be strong, and a strict finite and continuous $\mathcal{H}_{1}$-potential on $\Omega$, in [16] it is constructed a biharmonic space $(\Omega, \mathcal{H})$ whose harmonic spaces are $\left(\Omega, \mathcal{H}_{1}\right)$ and $\left(\Omega, \mathcal{H}_{2}\right)$. By using sections of continuous potentials, in [5] it is given a more general method for constructing such biharmonic space and it is proved a characterization of biharmonic spaces whose harmonic associated spaces are $\left(\Omega, \mathcal{H}_{1}\right)$ and $\left(\Omega, \mathcal{H}_{2}\right)$.

Here we prove that there is a unique biharmonic space $(\Omega, \mathcal{H})$ whose associated harmonic spaces are $\left(\Omega, \mathcal{H}_{1}\right)$ and $\left(\Omega, \mathcal{H}_{2}\right)$ with coupling kernel a given suitable kernel $V$ on $\Omega$.

Let $\omega$ be a relatively compact open subset of $\Omega$, we denote by $H_{\omega}^{1}(f)$ and $H_{\omega}^{2}(f)$ the solutions of the Dirichlet problem in $\omega$ with respect to the harmonic spaces $\left(\Omega, \mathcal{H}_{1}\right)$ and $\left(\Omega, \mathcal{H}_{2}\right)$ respectively, for the continuous boundary data $f$ on $\partial \omega$. We also denote the operators of reduction (resp. swept out) on $A \subset \Omega$ with respect to the harmonic sheafs $\mathcal{H}_{1}$ and $\mathcal{H}_{2}$ respectively by $R^{1, A}$ and $R^{2, A}$ (resp. $\widehat{R}^{1, A}$ and $\widehat{R}^{2, A}$ ).

The following result seems to be new in the axiomatic theory of biharmonic spaces:

Theorem 4.1. Let $\mathcal{H}_{1}$ and $\mathcal{H}_{2}$ be two Bauer harmonic sheaves on $\Omega$ and $V$ a Borel kernel on $\Omega$ such that for any $\varphi \in \mathcal{C}_{c}^{+}(\Omega)$, the function $V(\varphi)$ is a finite 
and continuous $\mathcal{H}_{1}$-potential $\mathcal{H}_{1}$-harmonic on $\Omega \backslash S(\varphi)$. Suppose moreover that $\Omega$ has a base $\mathcal{V}$ of open subsets which are both $\mathcal{H}_{1}$-regular and $\mathcal{H}_{2}$-regular, and that there exists a $\mathcal{H}_{2}$-potential $q>0$ such that $V q$ is a $\mathcal{H}_{1}$-potential. Then there exists a unique strong biharmonic space $(\Omega, \mathcal{H})$ whose associated harmonic spaces are $\left(\Omega, \mathcal{H}_{1}\right)$ and $\left(\Omega, \mathcal{H}_{2}\right)$, and $V$ as coupling kernel.

Remark 4.2. For a Borel kernel $V$ on an harmonic space $\Omega$, the property that for any continuous function $f \geq 0$ on $\Omega$ with compact support, the function $V f$ is a potential on $\Omega$, harmonic on $\Omega \backslash S(f)$ is equivalent to the property that for every $f \in \mathcal{B}^{+}(\Omega)$ which is bounded and equals zero outside a compact $K$ of $\Omega$, the function $V f$ is finite and continuous on $\Omega$ and harmonic on $\Omega \backslash K$.

Proof of Theorem 4.1. There exists a continuous $\mathcal{H}_{2}$-potential $0<q_{0} \leq q$, hence $V\left(q_{0}\right)$ is $\mathcal{H}_{1}$-superharmonic (because $q$ is the supremum of an increasing sequence of continuous potentials $q_{n}, n \in \mathbb{N}$ ). By replacing $q$ by $q_{0}$ we may assume that the $\mathcal{H}_{2}$-potential $q$ is continuous. For any $U \in \mathcal{U}_{c}$ we have $V(q)=V\left(1_{U} q\right)+V\left(1_{\Omega \backslash U} \cdot q\right)$. It follows from the hypotheses of the theorem that $V\left(1_{U} . q\right)$ is continuous on $\Omega$ and $V\left(1_{\Omega \backslash U} q\right)$ is $\mathcal{H}_{1}$-harmonic in $U$ (by Remark 4.2), hence $V(q)$ is continuous on $U$, and consequently $V(q)$ is continuous on $\Omega$. For any $U \in \mathcal{U}_{c}$, the potential (in $U$ )

$$
p_{U}=V(q)-\widehat{R}_{V(q)}^{1, \Omega \backslash U}=V(q)-H_{U}^{1}(V(q))
$$

is finite and continuous on $U$. The family $\left(p_{U}\right)_{U \in \mathcal{U}_{c}}$ is a section of continuous and finite $\mathcal{H}_{1}$-potentials on $\Omega$. Let $(\Omega, \mathcal{H})$ be the biharmonic space associated with the harmonic spaces $\left(\Omega, \mathcal{H}_{1}\right)$ and $\left(\Omega, \mathcal{H}_{2}\right)$ and the section of continuous potentials $\left(p_{U}\right)_{U \in \mathcal{U}_{c}}$ by [5].

It remains to show that the coupling kernel of $(\Omega, \mathcal{H})$ is equal to $V$. We may assume that the constant 1 is $\mathcal{H}_{2}$-harmonic on $\Omega$ and that $V 1$ is a $\mathcal{H}_{1}$ surharmonic, hence a $\mathcal{H}_{1}$-potential on $\Omega$. Let $V^{\prime}$ be the coupling kernel of biharmonic space $(\Omega, \mathcal{H})$. Since $V 1$ is a $\mathcal{H}_{1}$-potential and the pair $(V 1,1)$ is $\mathcal{H}$-harmonic in $\Omega$, then, according to the Corollary $3.10, V 1$ is the pure hyperharmonic function of order 2 associated to the constant function $u \equiv 1$, hence $V^{\prime} 1=V 1$. According to the conditions on $V$ and properties of the kernel $V^{\prime}$ we have $V=V^{\prime}$ following [8, Exercice 8.2.3., p. 198].

Let $(\Omega, \mathcal{H})$ be a strong biharmonic space with associated harmonic spaces $\left(\Omega, \mathcal{H}_{1}\right)$ and $\left(\Omega, \mathcal{H}_{2}\right)$, and suppose that the harmonic space $\left(\Omega, \mathcal{H}_{2}\right)$ is a Green space, and let $y \mapsto q_{y}$ be a continuous map from $\Omega$ into $\mathcal{S}_{2}(\Omega)$ endowed with the topology of R.-M. Hervé, which associates to every $y \in \Omega$ an $\mathcal{H}_{2}$-potential $q_{y}$ with harmonic support $\{y\}$, and denote by $G_{2}$ the function defined on $\Omega^{2}=\Omega \times \Omega$ by $G_{2}(x, y)=q_{y}(x)$. If for any $y \in \Omega$ the function $p_{y}^{\prime}$ (the pure hyperharmonic function associated with $q_{y}$ ) is $\mathcal{H}_{1}$-superharmonic, then the function $H$ defined on $\Omega^{2}$ by $H(x, y)=p_{y}^{\prime}(x)$ is called a (or the) biharmonic 
Green kernel or second Green kernel of the biharmonic space $(\Omega, \mathcal{H})$.

\section{EXAMPLES}

In this section we give some examples showing that the basic results of [18, 20] are incomplete. These results were used in 21] to define the adjoint biharmonic space of a Green biharmonic space. Although the results of [21] are true, they are built on results that seem to be incomplete as mentioned in the introduction and the previous sections. We shall clarify these aspects below.

Example 5.1. Let $N$ be an integer $\geq 1$, and $\left(\mathbb{R}^{N}, \mathcal{H}\right)$ the biharmonic space of the example 2.1 corresponding to $L_{1}=L_{2}=\Delta$. The space $(\Omega, \mathcal{H})$ is strong if and only if $N \geq 5$ (see for example [10, p. 588]). Suppose that $N \geq 5$ and let $(p, q)$ be a continuous $\mathcal{H}$-potential on $\mathbb{R}^{N}$ such that $p>0$ and $q>0$. The coupling kernel of $\left(\mathbb{R}^{N}, \mathcal{H}\right)$ is given by

$$
V f(x)=c_{N} \int_{\mathbb{R}^{n}} \frac{f(y)}{\|x-y\|^{N-2}} d \lambda(y)
$$

for any function $f \in \mathcal{B}_{+}\left(\mathbb{R}^{N}\right)$ and any $x \in \mathbb{R}^{N}$, where $\lambda$ denotes the Lebesgue measure on $\mathbb{R}^{N}$ and $c_{N}$ is a normalizing constant satisfying $\Delta \frac{c_{N}}{\|\cdot-y\|^{N-2}}=-\epsilon_{y}$ in the distribution sense for all $y \in \mathbb{R}^{N}$, and where $\epsilon_{y}$ is the Dirac measure at the point $y\left(G(x, y)=\frac{c_{N}}{\|x-y\|^{N-2}}\right.$ is the normalized Green kernel of $\left.\mathbb{R}^{N}\right)$. Note first that there is no biharmonic pair $(h, k)$ with $h, k>0$ on $\mathbb{R}^{N}$. Indeed, if a such pair exists, the function $k$ would be harmonic and $>0$ in $\mathbb{R}^{N}$, then constant, say $k=c>0$. We would then have $h(x) \geq V k(x)=c . c_{N} \int_{\mathbb{R}^{N}} \frac{1}{\|x-y\|^{N-2}} d \lambda(y)=$ $+\infty$ for any $x \in \mathbb{R}^{n}$, which is a contradiction.

Consider now the sheaf $\mathcal{H}^{\prime}$ of vector spaces of pairs of real continuous functions defined for any open subset $\omega$ of $\mathbb{R}^{N}$ by

$$
\mathcal{H}^{\prime}(\omega)=\{(h, k) \in \mathcal{C}(\omega) \times \mathcal{C}(\omega):(p h, q k) \text { is } \mathcal{H} \text {-biharmonic on } \omega\},
$$

so that we necessarily have

$$
\mathcal{H}^{\prime *}(\omega)=\left\{(u, v) \in \mathcal{C}_{l}(\omega) \times \mathcal{C}_{l}(\omega):(p u, q v) \text { is } \mathcal{H}-\text { hyperharmonic on } \omega\right\},
$$

where $\mathcal{C}_{l}(\omega)$ denotes the convex cone of nonnegative l.s.c. functions on $\omega$.

It is clear that $(1,1) \in \mathcal{H}^{\prime *}\left(\mathbb{R}^{N}\right)$. But there is no biharmonic pair $(u, v)$ on $\mathbb{R}^{N}$ such that $u>0$ and $v>0$. This example shows that the claims a) and b), in Remark 3 at page 323 of [20] are not true in general. 
Example 5.2. This example was given by the second author in [9] and [10]. Let $\Omega=[0,1[$ endowed by the topology induced by the usual one of $\mathbb{R}$. For any open subset $\omega$ of $\Omega$, we set

$$
\mathcal{H}_{1}(\omega)=\left\{u \in \mathcal{C}^{2}(\omega):(x u)^{\prime}=0\right\}
$$

and

$\mathcal{H}_{2}(\omega)=\left\{u \in \mathcal{C}(\omega): u(x)=\frac{a}{x^{2}}+b\right.$ on each connected component of $\left.\omega, a, b \in \mathbb{R}\right\}$,

where $\mathcal{C}(\omega)$, resp. $\mathcal{C}^{2}(\omega)$, denotes the space of continuous real functions, resp. real functions of class $\mathcal{C}^{2}$, on $\omega$ (if $0 \in \omega$ then each $u \in \mathcal{H}_{2}(\omega)$ is constant in the component of 0 ).

Then we define a biharmonic sheaf on $\Omega$ by putting

$$
\mathcal{H}(\omega)=\left\{(u, v) \in \mathcal{C}^{2}(\omega):(x u) "=-v, v \in \mathcal{H}_{2}(\omega)\right\}
$$

for any open subset $\omega$ of $\Omega$. It is easy to check that $(\Omega, \mathcal{H})$ is a Brelot biharmonic space whose associated harmonic spaces are $\left(\Omega, \mathcal{H}_{1}\right)$ and $\left(\Omega, \mathcal{H}_{2}\right)$.

Let $p$ and $q$ be the functions defined on $\Omega$ by $p(x)=\frac{1}{2}-\frac{x}{2}$ and $q(x)=$ $\min \left(1, v_{0}(x)\right)$ where

$$
v_{0}(x)=\left\{\begin{array}{cc}
\frac{1}{x^{2}}-1 & \text { if } x>0 \\
+\infty & \text { if } x=0 .
\end{array}\right.
$$

The function $v_{0}$ is an $\mathcal{H}_{2}$-potential. Indeed, $v_{0}$ is an $\mathcal{H}_{2}$-harmonic function on ]0, $1[$ and, for any $\alpha \in] 0,1\left[\right.$, if $k$ is a continuous function on $[0, \alpha], \mathcal{H}_{2}$-harmonic on $\omega=\left[0, \alpha\left[\right.\right.$ and such that $k(\alpha) \leq v_{0}(\alpha)$, then $k$ is a constant function on $[0, \alpha]$ and then we have $k \leq v_{0}$ on $\omega$. Hence $v_{0}$ is $\mathcal{H}_{2}$-superharmonic $>0$ on $\Omega$. Let $h$ be a $\mathcal{H}_{2}$-harmonic function on $\left[0,1\right.$ [ such that $h \leq v_{0}$, then $h$ is necessarily a constant function $\leq 0$, thus $v_{0}$ is an $\mathcal{H}_{2}$-potential.

One can also easily check that the pair $(p, q)$ is a $\mathcal{H}$-potential. Thus the biharmonic space $(\Omega, \mathcal{H})$ is strong. The harmonic spaces $\left(\Omega, \mathcal{H}_{1}\right)$ and $\left(\Omega, \mathcal{H}_{2}\right)$ are Green spaces, however, $\{0\}$ is not the biharmonic support of any (extremal) pure $\mathcal{H}$-potential. Indeed, $v_{0}$ is, up to the multiplication by some constant $>0$, the unique $\mathcal{H}_{2}$-potential of harmonic support $\{0\}$. If the pure hyperharmonic function $u$ of order 2 associated with $v_{0}$ is $\mathcal{H}_{1}$-superharmonic, then the pair $\left(u, v_{0}\right)$ would be $\mathcal{H}$-harmonic on ]0,1[according to the Proposition 3.8 , and then we would have $(x u) "(x)=-\frac{1}{x^{2}}+1$, hence necessarily

$$
u(x)=\frac{\ln (x)}{x}+\frac{x}{2}+a+\frac{b}{x},
$$

where $a$ and $b$ are a real constants. But this is impossible since $u(x)<0$ for $x$ close to 0. 
Let $(P, Q)$ be a continuous $\mathcal{H}$-potential on $\Omega$, with $Q>0$ (and then $P>0$ ) in $\Omega$. Consider the biharmonic sheaf $\mathcal{H}^{\prime}$ on $\Omega$ defined by

$$
\mathcal{H}^{\prime}(U)=\{(h, k) \in \mathcal{C}(U) \times \mathcal{C}(U):(P h, Q k) \in \mathcal{H}(U)\}
$$

for any open subset $U$ of $\Omega$. Then $(1,1) \in \mathcal{H}^{\prime *}(\Omega)$, but there is no pure $\mathcal{H}^{\prime}$ potential of biharmonic support $\{0\}$. This example shows that Theorem 2 of [20, p. 321] is not true in general.

Let $(\Omega, \mathcal{H})$ be a strong biharmonic space. We have seen that, unlike the harmonic spaces admitting a potential, the set of all points of $\Omega$ which are not the biharmonic support of any extremal pure potential is not always empty. In the case where $\left(\Omega, \mathcal{H}_{2}\right)$ is a Green harmonic space, it was shown in [18 that this set is nowhere dense (that is of empty interior) in $\Omega$ (cf. [18, Proposition 3.4]). In [10], the second author has shown the much more precise and general following result:

Theorem 5.3 ([10, Theorem 3.1]). Let $(\Omega, \mathcal{H})$ be a strong biharmonic space. Then the set of points of $\Omega$ which are not the biharmonic support of extremal pure potentials is $\mathcal{H}_{2}$-polar.

The proof of this result is based on the integral representation in the cone of nonnegative $\mathcal{H}$-superharmonic pairs (see [10]).

In 20] it is also studied the existence and the regularity of the biharmonic Green kernel ([20, Proposition 6]), however it seems also that his result is not true in general.

A Green harmonic space $(\Omega, \mathcal{K})$ is said to be symmetric if there is a continuous function $y \mapsto p_{y}$ from $\Omega$ onto the cone of $\mathcal{K}$-superharmonic functions $\geq 0$ endowed with the topology of R.-M. Hervé such that for every $y \in \Omega$, the function $p_{y}$ is a $\mathcal{K}$-potential of harmonic support $\{y\}$ and $p_{y}(x)=p_{x}(y)$ for any pair $(x, y) \in \Omega^{2}$ (then we say that the kernel-function $G(x, y)=p_{y}(x)$, the Green kernel of $(\Omega, \mathcal{K})$, is symmetric).

Theorem 5.4 ([10, Proposition 3.3]). Let $(\Omega, \mathcal{H})$ be a strong Green biharmonic space whose associated harmonic spaces $\left(\Omega, \mathcal{H}_{1}\right)$ and $\left(\Omega, \mathcal{H}_{2}\right)$ are symmetric and equal and, for any $y \in \Omega$, let $q_{y}$ be a $\mathcal{H}_{2}$-potential with harmonic support $\{y\}$. Then for any $y \in \Omega$, the pure hyperharmonic function $p_{y}^{\prime}$ of order 2 associated with $q_{y}$ is superharmonic. Moreover, if the function $G$ defined by $G(x, y)=q_{y}(x)$ is symmetric, then the function $H(x, y)=p_{y}^{\prime}(x)$ is symmetric.

If the harmonic spaces $\left(\Omega, \mathcal{H}_{1}\right)$ and $\left(\Omega, \mathcal{H}_{2}\right)$ are symmetric but not equal, the function $p_{y}^{\prime}$ is not always a $\mathcal{H}_{1}$-superharmonic function for any $y \in \Omega$, contrary to the claim in [20], which is not true in general as it is shown by the following example: 
Example 5.5. Let $(\Omega, \mathcal{H})$ be the biharmonic space of the example 5.2. For any $y \in \Omega$ consider the functions

$$
p_{y}(x)=\left\{\begin{array}{l}
\frac{1}{y}-1 \text { if } 0 \leq x \leq y \\
\frac{1}{x}-1 \text { if } y \leq x<1
\end{array}\right.
$$

and

$$
q_{y}(x)=\left\{\begin{array}{l}
\frac{1}{y^{2}}-1 \text { if } 0 \leq x \leq y \\
\frac{1}{x^{2}}-1 \text { if } y \leq x<1,
\end{array}\right.
$$

with the convention $\frac{1}{0}=+\infty$. Then, for each $y \in \Omega$, every $\mathcal{H}_{1}$-potential, resp. $\mathcal{H}_{2}$-potential, of harmonic support $\{y\}$ is proportional to $p_{y}$, resp. $q_{y}$. Put $G_{1}(x, y)=p_{y}(x)$ and $G_{2}(x, y)=q_{y}(x)$ for any pair $(x, y) \in \Omega^{2}$. The functions $G_{1}$ and $G_{2}$ are continuous on $\Omega \times \Omega$ and, for any $y \in \Omega$ and any $i=1,2$, the function $G_{i}(., y)$ is an $\mathcal{H}_{i}$-potential $\mathcal{H}_{i}$-harmonic on $\Omega \backslash\{y\}$. Thus $G_{1}$ and $G_{2}$ are (the) Green kernels of the harmonic spaces $\left(\Omega, \mathcal{H}_{1}\right)$ and $\left(\Omega, \mathcal{H}_{2}\right)$ respectively. It is clear that $G_{1}$ and $G_{2}$ are symmetric. However the pure hyperharmonic function of order 2 associated with $q_{0}$ is identically equal to $+\infty$. This example shows also that if the spaces $\left(\Omega, \mathcal{H}_{1}\right)$ and $\left(\Omega, \mathcal{H}_{2}\right)$ are symmetric but not necessarily equal, the biharmonic Green kernel is not necessarily defined.

\section{ThE ADJoInt BIHARMONIC SPACE}

The notion of adjoint harmonic functions in a Green harmonic space was introduced and studied by R.-M. Hervé in [14, Chap. VI] to which we refer for the definition and the properties of these functions.

An attempt to consider and study the adjoint theory for biharmonic functions was made in [21]. However, the results of [21] are based on incomplete results as we have pointed out through the examples 5.1 and 5.2. In this section we shall show that we can define correctly and precisely a theory of adjoint biharmonic space of a Green biharmonic space $(\Omega, \mathcal{H})$ in the case where this space has a "regular" biharmonic Green kernel (condtion (6.3) below). We shall also show that this last condition is necessary for the existence of the biharmonic adjoint of $(\Omega, \mathcal{H})$.

The incomplete construction of the adjoint biharmonic space in [21] is based on the Theorem 2 of [20] which is not true in general according to the example 5.2 of Section 5. To define correctly the adjoint biharmonic space, we shall proceed in a different way.

In this section $(\Omega, \mathcal{H})$ is a Green biharmonic space and the cône $\mathcal{S}_{j}^{+}(\Omega)$, $j=1,2$, is equipped with the topology of R.-M. Hervé (see [14, Chap. IV]). For any $y \in \Omega$, we consider an $\mathcal{H}_{1}$-potential $p_{y}$ and an $\mathcal{H}_{2}$-potential $q_{y}$ of harmonic support $\{y\}$ such that the functions $y \mapsto p_{y}$ and $y \mapsto q_{y}$ from $\Omega$ into $\mathcal{S}_{1}^{+}(\Omega)$ and $\mathcal{S}_{2}^{+}(\Omega)$ respectively are continuous (cf. [14, Theorem 18.1 and Proposition 
18.1, p.p. 479-480]), and we denote by $p_{y}^{\prime}$ the pure $\mathcal{H}_{1}$-hyperharmonic function associated with $q_{y}$.

Proposition 6.1. The following assertions are equivalent:

1. The map $y \mapsto p_{y}^{\prime}$ is continuous on $\Omega$.

2. For any $x \in \Omega$, the function $y \mapsto p_{y}^{\prime}(x)$ is continuous on $\Omega \backslash\{x\}$.

Proof. $1 . \Rightarrow 2$. Suppose that the map $y \mapsto p_{y}^{\prime}$ is continuous on $\Omega$ and let $x \in \Omega$ and $\left(y_{n}\right)$ a sequence of points $\Omega \backslash\{x\}$ converging to $y \in \Omega \backslash\{x\}$. Then we have $\operatorname{lim\operatorname {inf}} p_{y_{n}}^{\prime}=p_{y}^{\prime}$ and $\lim \widehat{\inf } q_{y_{n}}=q_{y}$, hence there exists $x_{0} \in \Omega \backslash\{y\}$ and a subsequence $\left(y_{n_{k}}\right)$ of $\left(y_{n}\right)$ such that $\lim p_{y_{n_{k}}}^{\prime}\left(x_{0}\right)=p_{y}^{\prime}\left(x_{0}\right)$ and $\lim q_{y_{n_{k}}}\left(x_{0}\right)=$ $q_{y}\left(x_{0}\right)$. Then it follows from [19, Proposition 1.8, p. 301] and Ascoli-Arzela's Theorem that there is a subsequence $\left(p_{j}, q_{j}\right)$ of $\left(\left(p_{y^{\prime}, n_{k}}, q_{n_{k}}\right)\right)$ which converges locally uniformly on $\Omega \backslash\{y\}$ to an $\mathcal{H}$-superharmonic pair $\left(p, q_{y}\right)$, where $p$ is a continuous function on $\Omega \backslash\{y\}$. Hence $p=\lim \widehat{\inf } p_{j}$ on $\Omega \backslash\{y\}$ and therefore on $\Omega$. Since the pair $\left(\lim \widehat{\inf } p_{j}, q_{y}\right)$ is $\mathcal{H}$-superharmonic on $\Omega$, we have $p \geq p_{y}^{\prime}$ on $\Omega \backslash\{y\}$. It follows that $p=p_{y}^{\prime}$ since $p-p_{y}^{\prime}$ is a nonnegative $\mathcal{H}_{1}$-superharmonic on the domain $\Omega \backslash\{y\}$ and vanishes at $x_{0}$. In particular we have $p(x)=p_{y}^{\prime}(x)$, so that $\lim p_{j}(x)=p_{y}^{\prime}(x)$. It follows that for any $x \in \Omega \backslash\{y\}$, the sequence $\left(p_{y_{n}}^{\prime}(x)\right)$ converges to $p_{y}^{\prime}(x)$. This proves the assertion 2 .

$2 . \Rightarrow 1$. Let $x \in \Omega$ and suppose that the function $y \mapsto p_{y}^{\prime}(x)$ is continuous on $\Omega \backslash\{x\}$. Let $\left(y_{n}\right)$ be a sequence of points of $\Omega$ converging to $y$ and $\left(y_{n_{k}}\right)$ a subsequence of $\left(y_{n}\right)$ such that the sequence $\left(p_{y_{n_{k}}}^{\prime}\right)$ converges in $\mathcal{S}_{1}^{+}(\Omega)$, we have $\lim \inf p_{y_{n_{k}}}^{\prime}(x)=p_{y}(x)$. On the other hand the pair $\left(\lim \widehat{\inf } p_{y_{n_{k}}}^{\prime}, q_{y}\right)$ is $\mathcal{H}$ hyperharmonic on $\Omega$, hence $\lim \widehat{\inf } p_{y_{n_{k}}}^{\prime} \geq p_{y}^{\prime}$. It follows then that $\lim \widehat{\inf } p_{y_{n_{k}}}^{\prime}=$ $p_{y}^{\prime}$ on $\Omega \backslash\{x\}$, hence on all of $\Omega$. We conclude that the map $\mapsto p_{y}^{\prime}$ is continuous on $\Omega$.

The next proposition and its corollary give some concrete conditions under which the map $y \mapsto p_{y}^{\prime}$ is continuous.

Definition 6.2. A (function) kernel on $\Omega$ is a l.s.c. function $G: \Omega \times \Omega \rightarrow$ $[0,+\infty]$. The kernel $G$ on $\Omega$ is said to be regular if, for any measure $\mu$ on $\Omega$ with compact support $K$, the function $G \mu$ is continuous on $\Omega$ if its restriction to $K$ is continuous.

In a Green harmonic space $(X, \mathcal{K})$, it follows by [14, p. 521 and Théorème 25.1 , p. 522], that the Green kernel of $X$ is regular if and only if $(X, \mathcal{K})$ satisfies the axiom $\mathrm{D}$ (axiom of domination, see [14, Chapitre V]).

In the following we denote by $G$ the kernel defined on $\Omega \times \Omega$ by $G(x, y)=$ $p_{y}(x)$. 
Proposition 6.3. Suppose that for any $y \in \Omega$, the function $p_{y}^{\prime}$ associated with $q_{y}$ is superharmonic, and that the kernel $G$ is regular. Then the map $y \mapsto p_{y}^{\prime}$ is continuous from $\Omega$ into $\mathcal{S}_{1}^{+}(\Omega)$.

Proof. We may suppose that $(1,1)$ is a pure $\mathcal{H}$-potential. By Proposition 3.4 the coupling kernel $V$ is given by $V f(x)=\int G(x, y) f(y) d \mu(y)$ for any $f \in \mathcal{B}^{+}(\Omega$ and $x \in \Omega$, where $\mu$ is the (unique) Radon measure $\geq 0$ on $\Omega$ such that $1=\int G(\cdot, y) d \mu(y)$. Let $\left(y_{n}\right)$ be a sequence of points of $\Omega$ converging to $y \in \Omega$. For any $\varphi \in \mathcal{C}_{c}^{+}(\Omega)$, the function $V(\varphi)$ is continuous, hence the sequence of measures $q_{y_{n}} \cdot \mu$ on $\Omega$ converges weakly to $q_{y} \cdot \mu$. Now we have $p_{y_{n}}^{\prime}=G \mu_{n}$ for every integer $n$, and hence $\lim p_{y_{n}}^{\prime}=\lim \widehat{\inf } G \mu_{n}=G \mu=p_{y}^{\prime}$ by [7, Theorem 3', p. 40].

Corollary 6.4. If the harmonic space $\left(\Omega, \mathcal{H}_{2}\right)$ satisfies the axiom of domination (or Axiom D, see [14, Chapitre V]), then

1. The map $y \mapsto p_{y}^{\prime}$ from $\Omega$ into $\mathcal{S}_{2}^{+}(\Omega)$ is continuous.

2. For any $x \in \Omega$, the function $y \mapsto p_{y}^{\prime}(x)$ is continuous on $\Omega \backslash\{x\}$.

Proof. If the harmonic space $\left(\Omega, \mathcal{H}_{2}\right)$ satisfies the axiom D, then kernel $G$ is regular as mentioned above, hence the assertion 1. and 2. hold according to Proposition 6.1 and Proposition 6.3,

To consider an adjoint space, we shall proceed differently to [21]. However, to do this, we need supplementary assumptions on the given biharmonic space $(\Omega, \mathcal{H})$ :

6.1. There is a base $\mathcal{V}$ of open subsets of $\Omega$ that are $\mathcal{H}_{i}$-completely determining for all $i=1,2$ (see Definition of completely determining open subset of a Brelot harmonic space in [14, no 11, p. 449]).

6.2. We suppose that the pair $(1,1)$ is a finite and continuous $\mathcal{H}$-potential (which is not really a restriction since we can always reduce the situation to this case by considering the $(f, g)$-harmonic pairs, where $(f, g)$ is a continuous $\mathcal{H}$-potential pair $>0$ on $\Omega$ ).

Let $V$ be the coupling kernel of $(\Omega, \mathcal{H})$. We denote by $\mu$ the measure representing the pure hyperharmonic function $V 1$ of order 2 associated with the $\mathcal{H}_{2}$-superharmonic constant function which equals 1 , that is:

$$
V 1=\int p_{y} d \mu(y)
$$

We recall that, according to the Proposition 3.11, the kernel $V$ is given by

$$
V f(x)=\int p_{y}(x) f(y) d \mu(y), \forall x \in \Omega \text { and } \forall f \in \mathcal{B}_{+}(\Omega) .
$$

We shall couple the biharmonic spaces $\left(\Omega,{ }^{*} \mathcal{H}_{2}\right)$ and $\left(\Omega,{ }^{*} \mathcal{H}_{1}\right)$ so that in the obtained biharmonic space, the function $p^{\prime}(y)$ is the pure hyperharmonic 
function of order 2 associated with the ${ }^{*} \mathcal{H}_{1}$-potential $p_{y}^{*}$ for any $y \in \Omega$. To do this we shall use the coupling defined in the proof of Theorem 4.1 relatively to the kernel $V^{*}$ defined by

$$
V^{*} f(x)=\int q_{x}(y) f(y) d \mu(y), \forall f \in \mathcal{B}_{+}(\Omega)
$$

so that we have, in particular,

$$
V^{*}\left(p_{y}^{*}\right)(x)=V^{*}(p .(y))(x)=\int p_{x}(z) q_{z}(y) d \mu(z)=p_{x}^{\prime}(y), \forall x, y \in \Omega .
$$

The kernel $V^{*}$ will be called the "adjoint" kernel associated with $V$.

The conditions 6.1, 6.2 mean that the kernel $V^{*}$ satisfies some of the hypotheses of Theorem 4.1. In order $V^{*}$ is the coupling kernel, it needs to satisfy the following condition:

6.3. For any function $\varphi \in \mathcal{C}_{c}^{+}(\Omega)$, the function $V^{*} \varphi$ is finite and continuous on $\Omega$.

We do not know if the condition 6.3. is always satisfied (under the hypotheses of the present section). However we have the following

Proposition 6.5. If for any $y \in \Omega$, the function $p_{y}^{\prime}$ is superharmonic and if the function $y \mapsto p_{y}^{\prime}$ from $\Omega$ into $\mathcal{S}_{2}^{+}(\Omega)$ is continuous, then the condition $\mathbf{6 . 3}$ is satisfied.

Proof. Let $\varphi \in \mathcal{C}_{c}^{+}(\Omega), \varphi \not \equiv 0$, and $y \notin S(\varphi)$, the support of $\varphi$. Since the function $p_{y}^{*}$ is continuous and $>0$ on $S(\varphi)$, we can find $\alpha \geq 0$ such that $\varphi \leq \alpha p_{y}^{*}$, and therefore $\alpha p^{\prime}(y)=V^{*}\left(\alpha p_{y}^{*}-\varphi\right)+V^{*}(\varphi)$. Since the function $p^{\prime}(y)$ is finite and continuous on a neighborhood $U$ of $S(\varphi)$ not containing $y$ (following Proposition 6.1), and that $V^{*}\left(\alpha p_{y}^{*}-\varphi\right)$ and $V^{*}(\varphi)$ are l.s.c., then $V^{*}(\varphi)$ is finite and continuous on $U$. On the other hand, we have $V^{*} \varphi(x)=$ $\int q_{y}^{*}(x) \varphi(y) d \mu(y)$, and therefore $V^{*} \varphi$ is ${ }^{*} \mathcal{H}_{2}$-harmonic on the complement of the support $\varphi$. Hence $V^{*} \varphi$ is finite and continuous on $\Omega$.

Corollary 6.6. If the harmonic space $\left(\Omega, \mathcal{H}_{2}\right)$ satisfies the axiom of domination then the condition $\mathbf{6 . 3}$ is satisfied.

Proof. The corollary results easily from the above proposition and Corollary 6.4 .

One may ask if the condition $\mathbf{6 . 3}$ is satisfied by $V^{*}$ in the case where there are points of $\Omega$ which are not the biharmonic support of a pure potential. The example 5.2 shows that the answer is not true in general. To see this (in the example 5.2) note first that $\left(\frac{1-x}{2}, 1\right)$ is a finite continuous $\mathcal{H}$-superharmonic 
pair on $[0,1[$. For any $y \in[0,1[$ we put

$$
p_{y}(x)=\left\{\begin{array}{l}
\frac{1}{y}-1 \text { if } 0 \leq x \leq y \\
\frac{1}{x}-1 \text { if } y \leq x<1
\end{array}\right.
$$

and

$$
q_{y}(x)=\left\{\begin{array}{l}
\frac{1}{y^{2}}-1 \text { if } 0 \leq x \leq y \\
\frac{1}{x^{2}}-1 \text { if } y \leq x<1,
\end{array}\right.
$$

with the convention $\frac{1}{0}=+\infty$. It is clear that the functions $y \mapsto p_{y}$ and $y \mapsto q_{y}$ from $\Omega$ into $\mathcal{S}_{1}^{+}(\Omega)$ and from $\Omega$ into $\mathcal{S}_{2}^{+}(\Omega)$ respectively, endowed with the topology of R.-M. Hervé, are continuous. Let $\mu$ be the measure on $\Omega$ defined by $\mu(d y)=y(1-y) \lambda(d y)$, where $\lambda$ denotes the Lebesgue measure on $[0,1[$. A simple calculation which we do not detail here gives $\frac{1}{2}(1-x)=\int p_{y}(x) d \mu(y)$. The coupling kernel of the harmonic spaces $\left(\Omega, \mathcal{H}_{1}\right)$ and $\left(\Omega, \mathcal{H}_{2}\right)$ in this case is given by

$$
V f(x)=\int p_{y}(x) f(y) d \mu(y)
$$

and thus

$$
V^{*} f(x)=\int q_{x}(y) f(y) d \mu(y)
$$

for any function $f \in \mathcal{B}^{+}(\Omega)$ and any $x \in \Omega$. Let $\varphi$ be a nonnegative continuous on $[0,1$ of compact support and such that $\varphi(0)>1$. Then we can find a real $\alpha \in] 0,1[$ such that $\varphi \geq 1$ on $[0, \alpha]$ so we have

$$
V^{*}(\varphi)(0) \geq \int_{0}^{\alpha} \frac{\left(1-y^{2}\right)(1-y)}{y} d y=+\infty,
$$

which proves that the condition $\mathbf{6 . 3}$ is not satisfied. We shall prove later (Proposition 6.9 ) the converse of Proposition 6.5, that is, if the condition 6.3 (of course in addition of $\mathbf{6 . 1}, \mathbf{6 . 2}$ ) is satisfied, then the function $p_{y}^{\prime}$ is $\mathcal{H}_{1-}$ superharmonic for any $y \in \Omega$ and that the map $y \mapsto p_{y}^{\prime}$ is continuous.

Now we are going to couple the harmonic spaces $\left(\Omega,{ }^{*} \mathcal{H}_{2}\right)$ and $\left(\Omega,{ }^{*} \mathcal{H}_{1}\right)$ by mean of the kernel $V^{*}$, so that in the obtained biharmonic space, the function $p^{\prime}(y)$ is the pure hyperharmonic function of order 2 associated with the ${ }^{*} \mathcal{H}_{1^{-}}$ superharmonic function $p_{y}^{*}=p$. $(y)$ for any $y \in \Omega$ as we have seen above.

Theorem 6.7. Let $(\Omega, \mathcal{H})$ be a strong Green biharmonic space whose associated harmonic spaces $\left(\Omega, \mathcal{H}_{1}\right)$ and $\left(\Omega, \mathcal{H}_{2}\right)$ satisfy the conditions $\mathbf{6 . 1}, \mathbf{6 . 2}$ and 6.3. Then there is a unique (Green) biharmonic space $\left(\Omega,{ }^{*} \mathcal{H}\right)$ whose associated harmonic spaces are respectively $\left(\Omega,{ }^{*} \mathcal{H}_{2}\right)$ and $\left(\Omega,{ }^{*} \mathcal{H}_{1}\right)$ and such that for any $y \in \Omega$, the pure hyperharmonic of order 2 associated with $p_{y}^{*}$ is equal to $p^{\prime}(y)$. 
Proof. The kernel $V^{*}$ satisfies the hypotheses of Theorem 4.1, hence the existence (and uniqueness) of a biharmonic space $\left(\Omega,{ }^{*} \mathcal{H}\right)$ defined by the coupling of the harmonic spaces $\left(\Omega,{ }^{*} \mathcal{H}_{2}\right)$ and $\left(\Omega,{ }^{*} \mathcal{H}_{1}\right)$ by mean of this kernel. According to the formula (6.1), for any $y \in \Omega$ the pure hyperharmonic function of order 2 associated with the ${ }^{*} \mathcal{H}_{1}$-superharmonic function $p_{y}^{*}$ is equal to $p^{\prime}$. $(y)$.

The biharmonic space $\left(\Omega,{ }^{*} \mathcal{H}\right)$ in Theorem 6.7 is called the adjoint biharmonic space of $(\Omega, \mathcal{H})$. For any open set $\omega \subset \Omega$, the pairs $(u, v) \in \mathcal{H}^{*}(\omega)$ are called the adjoint biharmonic pairs on $\omega$.

Remark 6.8. The relatively compact completely determining open subsets of the base $\mathcal{V}$ form a base of open subsets which are both ${ }^{*} \mathcal{H}_{1}$-regular and ${ }^{*} \mathcal{H}_{2^{-}}$ regular, hence ${ }^{*} \mathcal{H}$-regular. Let $\omega$ be a ${ }^{*} \mathcal{H}$-regular (relatively compact) open subset and $\varphi$ a continuous function $\geq 0$ on $\partial \omega$. For any $x \in \omega$, the linear form $\varphi \mapsto V_{\omega}^{*}\left({ }^{*} H_{\omega}^{1}(\varphi)\right)(x)$ on $\mathcal{C}(\partial \omega)$ defines a (Radon) measure $\tau_{x}^{\omega}$ on $\partial \omega$. Here ${ }^{*} H_{\omega}^{1}(\varphi)$ is the solution of the Dirichlet problem in $\omega$ in the harmonic space $\left(\Omega,{ }^{*} \mathcal{H}_{1}\right)$ for the data $\varphi$ on $\partial \omega$, and $V_{\omega}$ is is the Borel kernel defined on $\omega$ by $V_{\omega}(f)=V(\bar{f})-\widehat{R}_{V(\bar{f})}^{1, \Omega \backslash \omega}$ for any $f \in \mathcal{B}^{+}(\omega)$, where $\bar{f}$ is the function on $\Omega$ which equals $f$ on $\omega$ and 0 on $\Omega \backslash \omega$. The triple of biharmonic measures of $\omega$ at the point $x$ is $\left(\rho_{x}^{\omega}, \tau_{x}^{\omega}, \sigma_{x}^{\omega}\right)$, where $\rho_{x}^{\omega}$ and $\sigma_{x}^{\omega}$ are respectively the harmonic measures of $\omega$ at the point $x$ in the adjoint harmonic spaces $\left(\Omega,{ }^{*} \mathcal{H}_{2}\right)$ and $\left(\Omega,{ }^{*} \mathcal{H}_{1}\right)$ (see [14, Chap. VI]).

Now we prove the converse of the Proposition 6.5.

Proposition 6.9. Suppose that there exists a strong biharmonic space $(\Omega, \mathcal{G})$ whose associated harmonic spaces are $\left(\Omega,{ }^{*} \mathcal{H}_{2}\right)$ and $\left(\Omega,{ }^{*} \mathcal{H}_{1}\right)$ and such that for any $y \in \Omega$, the function $p^{\prime}(y)$ is the pure hyperharmonic function of order 2 associated with $p_{y}^{*}=p .(y)$. Then for any $y \in \Omega$, the function $p_{y}^{\prime}$ is $\mathcal{H}_{1}$ superharmonic and the function $y \mapsto p_{y}^{\prime}$ is continuous on $\Omega$.

Proof. Suppose that there exists a strong biharmonic space $(\Omega, \mathcal{G})$ whose associated harmonic spaces are $\left(\Omega,{ }^{*} \mathcal{H}_{2}\right)$ and $\left(\Omega,{ }^{*} \mathcal{H}_{1}\right)$ and such that for any $y \in \Omega$, the function $p^{\prime}(y)$ is the pure hyperharmonic function of order 2 associated with $p_{y}^{*}=p .(y)$. Assume that there exists $y \in \Omega$ such that $p_{y}^{\prime} \equiv+\infty$ on $\Omega$, then we have $p_{x}^{\prime *}(y)=p_{y}^{\prime}(x)=+\infty$ for any $x \in \Omega \backslash(N \cup\{y\})$, but this is absurd according to the Theorem [5.3, since for such $x$ we have $p_{x}^{\prime}(y)<+\infty$ (because the pair $\left(p^{\prime}(x), p_{x}^{*}\right)$ is $\mathcal{G}$-biharmonic on $\left.\Omega \backslash\{x\}\right)$, where $N$ is the ${ }^{*} \mathcal{H}_{1^{-}}$ polar subset of points $y$ of $\Omega$ such that the pure hyperharmonic function of order 2 associated with $p_{y}^{*}$ is identically equal to $+\infty$. Now let $x \in \Omega$, then the pair $\left(p^{\prime}(x), q^{*}\right)$ is $\mathcal{H}$-harmonic on $\Omega \backslash\{x\}$ according to Proposition 3.8 . and hence the function $p^{\prime}(x)$ is continuous on $\Omega \backslash\{x\}$. Let $z \in \Omega \backslash\{x\}$ and $\mathcal{F}$ an ultrafilter on $\Omega$ finer than the filter of neighborhoods of $z$, then we have $\lim \widehat{\inf }_{\mathcal{F}} p_{y}^{\prime}(x)=p_{z}^{\prime}(x)$, and it follows that this equality holds also for $z=x$ 
(indeed, if $u$ and $v$ are $\mathcal{K}$-hyperharmonic on $X$ in a harmonic space $(X, \mathcal{K})$ such that $u=v$ on $X \backslash\{x\}$ for some point $x \in X$, then $u=v$ ). It follows then that the function $y \mapsto p_{y}^{\prime}$ is continuous on $\Omega$. The proposition is proved.

Now we proceed to establish a converse of Theorem 6.7, that is, the existence of an adjoint biharmonic space $\left(\Omega, \mathcal{H}^{*}\right)$ associated with the biharmonic space $(\Omega, \mathcal{H})$ satisfying the hypotheses of Theorem 6.7 and the property that for any $y \in \Omega$, the pure hyperharmonic function of order 2 associated with $p_{y}^{*}$ is $p^{\prime}(y)$, implies that the coupling kernel of $(\Omega, \mathcal{G})$ is $V^{*}$, and hence $V^{*}$ necessarily satisfies the condition $\mathbf{6 . 3}$.

Theorem 6.10. Suppose that there exists a biharmonic space $(\Omega, \mathcal{G})$ whose associated harmonic spaces are respectively $\left(\Omega,{ }^{*} \mathcal{H}_{2}\right)$ and $\left(\Omega,{ }^{*} \mathcal{H}_{1}\right)$, such that, for any $y \in \Omega$, the pure hyperharmonic function of order 2 associated with $p_{y}^{*}$ is equal to $p^{\prime}(y)$. Then the coupling kernel of $(\Omega, \mathcal{G})$ is $V^{*}$.

Proof. Let $W$ be the coupling kernel of $(\Omega, \mathcal{G})$. According to the hypotheses of the theorem, the biharmonic space $(\Omega, \mathcal{G})$ is strong. For any $y \in \Omega$, we have $V^{*}\left(p_{y}^{*}\right)=W\left(p_{y}^{*}\right)=p^{\prime}(y)$ by definition of $V^{*}$ and $W$. Let $p$ be a ${ }^{*} \mathcal{H}_{1}$-potential $>0$ on $\Omega$ such that $W p$ is finite and continuous on $\Omega$ (such a potential exists by Corollary [3.6). According to [14, Theorem 18.2, p. 482], there exists a measure $\mu$ on $\Omega$ such that $p=\int p$. $(y) d \mu(y)$. Then by applying twice the Fubini theorem, we have for any $x \in \Omega$,

$$
\begin{aligned}
W p(x) & =\int p(z) W(x, d z)=\iint p_{y}^{*}(z) d \mu(y) W(x, d z) \\
& =\iint p_{y}^{*}(z) W(x, d z) d \mu(y)=\int W\left(p_{y}^{*}\right)(x) d \mu(y) \\
& =\int V^{*}\left(p_{y}^{*}\right)(x) d \mu(y)=V^{*} p(x),
\end{aligned}
$$

and hence $W p=V^{*} p$. Since $W$ satisfies the condition 2 of Theorem 3.3, we deduce that $V^{*}$ also satisfies this condition. Indeed, let $\varphi \in \mathcal{C}_{c}^{+}(\Omega) \alpha>0$ such that $\varphi \leq \alpha p$. Then $\alpha V^{*} p=V^{*}(\varphi)+V^{*}(\alpha p-\varphi)$. The functions $V^{*}(\varphi)$ and $V^{*}(\alpha p-\varphi)$ are both finite and l.s.c. and $V^{*} p$ is finite and continuous on $\Omega$, then $V^{*}(\varphi)$ is finite and continuous. Moreover, by the definition of the kernel $V^{*}$, the function $V^{*} \varphi$ is ${ }^{*} \mathcal{H}_{2}$-harmonic in $\Omega \backslash S(\varphi)$. Let $v \in{ }^{*} \mathcal{H}_{1}^{*+}(\Omega)$, then according to the above results we have $W v=\sup _{n} W(v \wedge n p)=\sup _{n} V^{*}(v \wedge n p)=V^{*} v$. It therefore follows from Theorem 3.3 that $W=V^{*}$.

We have at the same time proved (under the assumptions of Theorem 6.10) the following result:

Corollary 6.11. Under the hypotheses of the previous theorem, for any $\varphi \in$ $\mathcal{C}_{c}^{+}(\Omega)$, the function $V^{*} \varphi$ is a finite and continuous ${ }^{*} \mathcal{H}_{2}$-potential on $\Omega$. 


\section{Application to the Study of the Regularity of the BIHARMONIC GREEN KERNEL}

We keep the notations of the previous sections. Let $(\Omega, \mathcal{H})$ be a strong Green biharmonic space. For any $y \in \Omega$, we consider an $\mathcal{H}_{2}$-potential $q_{y}$ of harmonic support $\{y\}$ such that the function $y \mapsto q_{y}$ from $\Omega$ into $\mathcal{S}_{1}^{+}(\Omega)$ (endowed with the topology of R.-M. Hervé) is continuous (cf. [14, Theorem 18.1 and Proposition 18.1, p. 479-480]). Suppose that, for any $y \in \Omega$, the function $p_{y}^{\prime}$ is $\mathcal{H}_{1}$-superharmonic on $\Omega$, and that the map $y \mapsto p_{y}^{\prime}$ is continuous from $\Omega$ into $\mathcal{S}_{1}^{+}(\Omega)$ (hence for any $\varphi \in \mathcal{C}_{c}^{+}(\Omega)$, the function $V^{*} \varphi$ is a finite and continuous ${ }^{*} \mathcal{H}_{2}$-potential). Put $H(x, y)=p_{y}^{\prime}(x)$ for any pair $(x, y) \in \Omega^{2}$. The kernel $H$ was called the biharmonic Green kernel of the biharmonic space $(\Omega, \mathcal{H})$ in the Section 2.

Theorem 7.1. Suppose that the conditions (6.1) and (6.2) are satisfied and that for any $y \in \Omega$, the function $p_{y}^{\prime}$ is $\mathcal{H}_{1}$-superharmonic on $\Omega$ and, for any $x \in \Omega$, the function $y \mapsto p_{y}^{\prime}(x)$ is continuous on $\Omega \backslash\{x\}$. Then the kernel $H$ has the following properties

1. $H$ is l.s.c. on $\Omega \times \Omega$.

2. $H$ is continuous on $\Omega \times \Omega \backslash \Delta$, where $\Delta=\{(x, x): x \in \Omega\}$ is the diagonal of $\Omega \times \Omega$.

Proof. We may suppose that the pair $(1,1)$ is $\mathcal{H}$-superharmonic. The hypotheses of Theorem 4.1 are satisfied, and thus the adjoint biharmonic space $\left(\Omega,{ }^{*} \mathcal{H}\right)$ is well defined.

1. We have $H(x, y)=\int p_{z}(x) q_{y}(z) d \mu(z)$ for any pair $(x, y) \in \Omega^{2}$. On the other hand, for any $z \in \Omega$, the functions $(x, y) \mapsto p_{z}(x)$ and $(x, y) \mapsto q_{y}(z)$ are l.s.c. on $\Omega \times \Omega$, then it follows from the Fatou lemma that the function $H$ is l.s.c. on $\Omega \times \Omega$.

2. Let $\left(\left(x_{n}, y_{n}\right)\right)$ be a sequence of points of $\Omega \times \Omega \backslash \Delta$ which converges to $(x, y) \in \Omega \times \Omega \backslash \Delta$, and $\omega$ an open neighborhood of $x$ such that $y \notin$ $\bar{\omega}$. We can obviously assume that $y_{n} \notin \bar{\omega}$ for any integer $n$. The pairs $(H(x, \cdot), q .(x))$ are ${ }^{*} \mathcal{H}$-biharmonic, hence continuous on $\Omega \backslash\{x\}$. Thus the sequences $\left(H\left(x, y_{n}\right)\right)$ and $\left(q_{y_{n}}(x)\right)$ are bounded. On the other hand, the pairs $\left(H\left(., y_{n}\right), q_{y_{n}}\right), n \in \mathbb{N}$, are biharmonic on $\omega$, then, according to [10, Theorem 3.2 , p. 586], the sequences of functions $\left(H\left(., y_{n}\right)\right)_{n \in \mathbb{N}},\left(q_{y_{n}}\right)_{n \in \mathbb{N}}$, are equicontinuous at $x$. Let $\epsilon>0$, we can find a neighborhood $W$ of $x$ such that $W \subset \omega$ and $\left|H\left(\xi, y_{n}\right)-H\left(x, y_{n}\right)\right|<\epsilon$ for any $\xi \in W$ and any $n \in \mathbb{N}$. By applying the same arguments in he biharmonic space $\left(\Omega,{ }^{*} \mathcal{H}\right)$ to the kernel $H^{*}$ (defined by $\left.H^{*}(x, y)=H(y, x)\right)$, there is an open neighborhood $W^{\prime}$ of $y$ such that $\left|H\left(x_{n}, \xi\right)-H\left(x_{n}, y\right)\right|<\epsilon$ for any $\xi \in W^{\prime}$ and any $n \in \mathbb{N}$. Furthermore, there exists an integer $n_{0} \in \mathbb{N}$ such that $x_{n} \in W$ and $y_{n} \in W^{\prime}$ for any $n \geq n_{0}$. Thus

$$
\left|H\left(x_{n}, y_{n}\right)-H(x, y)\right| \leq\left|H\left(x_{n}, y_{n}\right)-H\left(x_{n}, y\right)\right|+\left|H\left(x_{n}, y\right)-H(x, y)\right|<2 \epsilon .
$$


for every $n \geq n_{0}$. We then deduce that the sequence $\left(H\left(x_{n}, y_{n}\right)\right)$ converges to $H(x, y)$. Hence the function $H$ is continuous on $\Omega \times \Omega \backslash \Delta$.

\section{REFERENCES}

[1] E.M. Alfsen, Compact Convex Sets and Boundary Integrals, Ergebnisse der Math. Vol. 57, Springer, Berlin, 2001.

[2] N. Boboc, Gh. Bucur, Perturbations in excessive structures. Complex analysis-fifth Romanian-Finnish seminar, Part 2 (Bucharest, 1981), 155-187, Lecture Notes in Math., 1014, Springer, Berlin, 1983.

[3] N. Bouleau, Espaces biharmoniques, systèmes d'équations différentielles et couplage de processus de Markov, J. Math. Pures Appl. 59 (1980), 187-240.

[4] N. Bouleau, Application de la théorie markovienne du potentiel 'a létude de fonctions biharmoniques et de certains systimes différentiels. et couplage de processus de Markov, Thèse dEtat de lUniversité Paris VI (Mars 1980).

[5] A. Boukricha, Espaces biharmoniques. Théorie du potentiel (Orsay, 1983), 116-148, Lecture Notes in Math., 1096, Springer, Berlin, 1984.

[6] Boboc, N., Bucur, Gh., Cornea, A.: Order and Convexity in Potential Theory: HCones, Lecture Notes in Math. 853, Springer, Berlin, 1981.

[7] Brelot, M.: Lectures on Potential theory, 2d Edition, Tata Institute of Fundamental Research, Bombay, 1967.

[8] C. Constantinescu, A. Cornea, Potential Theory on Harmonic spaces, Springer Verlag Heidelberg, 1972.

[9] M. El Kadiri, Représentation intégrale dans le cadre de la théorie axiomatique des fonctions biharmoniques, Thèse de 3e cycle, Rabat, 1986.

[10] M. El Kadiri, Représentation intégrale en théorie des fonctions biharmoniques, Rev. Roumaine Math. Pures Appl., Tome XLII, nos 7-8 (1997), 579-589.

[11] M. El Kadiri, Frontière de Martin biharmonique et représentation intégrale des fonctions biharmoniques positives, Positivity 6, no 2 (2002), 129-145.

[12] M. El Kadiri, S. Haddad, Remarques sur la frontière de Martin biharmonique et représentation intégrale des fonctions biharmoniques positives, Int. J. Math. Math. Sci., no. 9 (2005), 1461-1472.

[13] L. L. Helms, Introduction to potential theory, Pure and Applied Mathematics, Vol. XXII, Wiley-Interscience, New York-London-Sydney 1969.

[14] R-M. Hervé, Recherches axiomatiques sur la théorie des fonctions surharmoniques et du potentiel. Ann. Inst. Fourier (Grenoble) 12 (1962), 415-571.

[15] P-A. Meyer, Brelot's axiomatic theory of the Dirichlet problem and Hunt's theory. Ann. Inst. Fourier (Grenoble) 13, fasic. 2 (1963), 357-372.

[16] E.P. Smyrnelis, Axiomatique des fonctions biharmoniques, Section 1, Ann. Inst. Fourier 25 (1975), 35-97.

[17] E.P. Smyrnelis, Axiomatique des fonctions biharmoniques, Section 2, Ann. Inst. Fourier 26 (1976), 1-47.

[18] E.P. Smyrnelis, Représentation intégrale dans les espaces biharmoniques, Acad. Roy. Belg. Bull. Cl. Sci. 71 (1985), 383-394.

[19] E.P. Smyrnelis, Harnack's properties of biharmonic functions, Comment. Math. Univ. Carolin. 33 (1992), no. 2, 299-302.

[20] E.P. Smyrnelis, Couples de Green, Acad. Roy. Belg. Bull. Cl. Sci. 12 (2002), 319-326. 
[21] E.P. Smyrnelis, Fonctions biharmoniques adjointes, Ann. Pol. Math. 99, no 1 (2010), $1-21$.

University of Mohammed V, Department of Mathematics, Faculty of SciEnces, P.B. 1014, Rabat, Morocco

E-mail address: slimonier.math@gmail.com

Université Mohammed V, Département de Mathématiques, Faculté des SciENCES, B.P. 1014, Rabat, Morocco

E-mail address: el.imad.imad@gmail.com

E-mail address: elkadiri30@hotmail.com

CRMEF, RABAT-AKkari, Morocco

E-mail address: sabahhaddad24@gmail.com 